\title{
Mucocele of the middle turbinate: A rare entity
}

\author{
Nitin Gupta, Madhur Kalia, Arjun Dass, Surinder K Singhal
}

Department of Ear, Nose \& Throat Government Medical College and Hospital, Chandigarh, India

\section{ABSTRACT}

BACKGROUND. Mucocele is a benign expansile cyst-like lesion seen in the paranasal sinuses, which has a tendency to expand and erode the surrounding sinus walls. These mucoceles develop as a result of obstruction of the sinus ostium and superadded infection. As such, the frontoethmoidal area is the most common site, followed by the maxillary sinus and the sphenoid sinus. Rarely, mucocele may also develop in abnormally aerated bones, such as middle turbinate (concha bullosa), clinoid process and pterygoid process.

CASE REPORT. We report two infrequent cases of mucocele of concha bullosa, clinically presenting as the cause of nasal obstruction, which were completely removed endoscopically, and patients were symptomatically relieved.

CONCLUSION. Mucocele of the middle turbinate represents a diagnostic challenge to surgeons both in terms of symptoms and risk of complications. Therefore, the condition should be considered as a possible cause of progressive nasal obstruction by otolaryngologists, and careful examination of the nasal cavity is necessary to determine the existence of this rare condition.

KEYWORDS: mucocele, paranasal sinus, concha bullosa.

\section{INTRODUCTION}

Mucoceles are respiratory epithelium lined cystic lesions found in the paranasal sinuses. Most commonly seen in the frontoethmoidal area, these mucoceles develop as a result of obstruction of the normal sinus drainage tract with superadded infection. Inflammatory mediators are secreted, which lead to bone resorption and expansion of the mucocele. Computed tomography (CT) scan shows an expansile homogenously iso- to hyperdense mass filling the sinusal cavity ${ }^{1}$.

Concha bullosa is one of the most common anatomical variations in the nasal cavity. It refers to an air-filled cavity within the middle turbinate. Obstruction of the concha bullosa can rarely lead to formation of a mucocele which may be secondarily infected forming a mucopyocele. The mucocele of the middle turbinate may present as uncommon cause of nasal obstruction, headache and other nasal or ocular symptoms, which, if correctly diagnosed, can be completely reversed by endoscopic surgery alone ${ }^{2}$.

In this article we present two such cases of muco- cele of the middle turbinate presenting as uncommon cause of nasal obstruction, which were completely endoscopically resected.

\section{CASE REPORT}

\section{CASE 1}

A 14-year-old male patient presented with a sixmonth history of bilateral nasal obstruction, predominantly on the right side, which was gradually progressive and associated with mucus nasal discharge. The patient also complained of right-sided frontal headache. There was no history suggestive of allergic rhinitis. Past medical history was unremarkable. Anterior rhinoscopy revealed gross deviated nasal septum to the left side. On the right nasal fossa, there was an enlarged middle turbinate suggestive of concha bullosa. Diagnostic nasal endoscopy revealed an enlarged middle turbinate occupying almost the entire right nasal cavity, pushing the nasal septum to the left side.

Computerized tomography (Figure $1 \mathrm{~A}, \mathrm{~B}$ ) of the nose and paranasal sinuses revealed heterogenous 

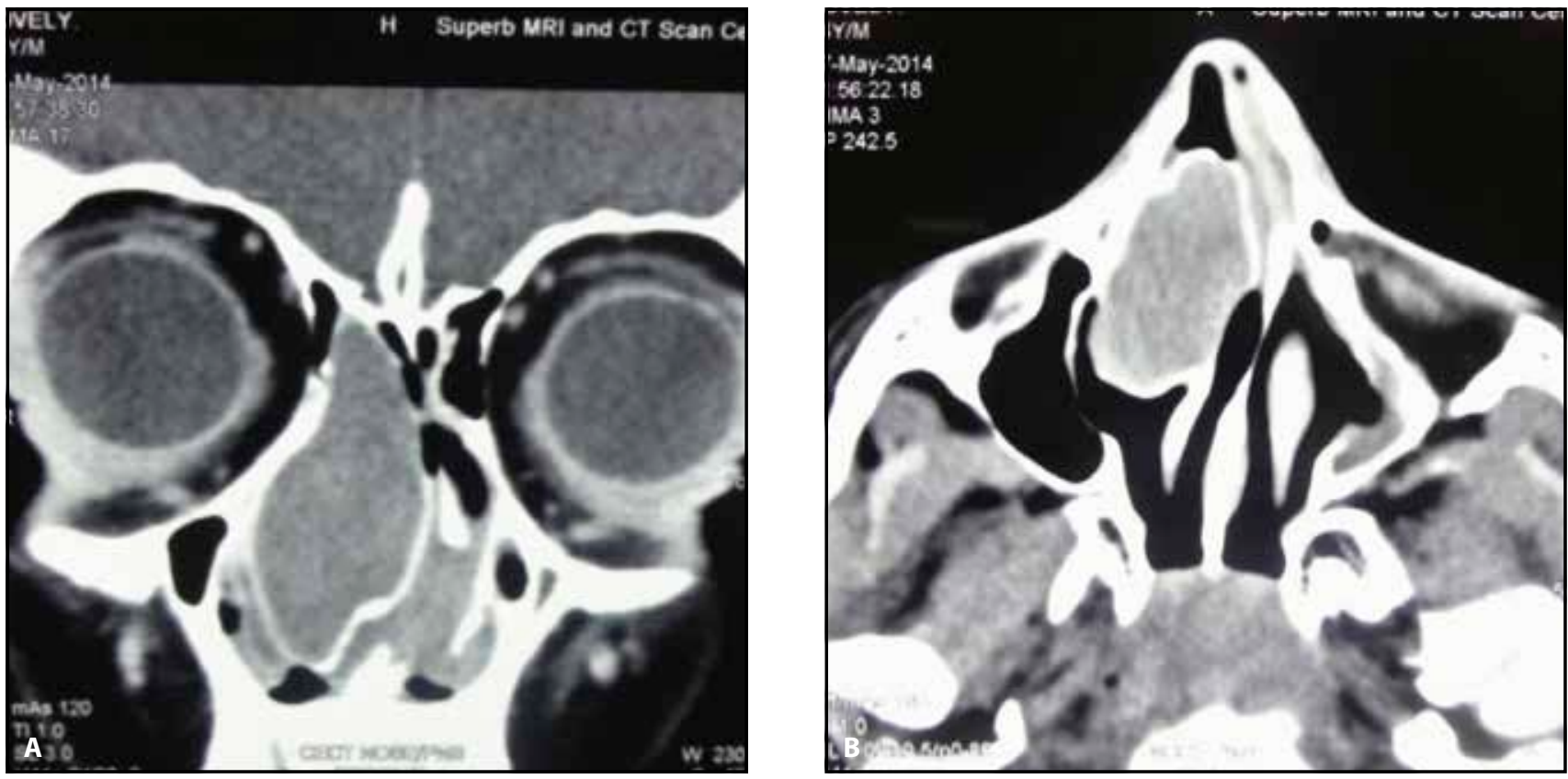

Figure 1. Coronal (A) and axial (B) sections of computed tomography of a patient with right-sided concha bullosa with heterogenous soft tissue density within, suggestive of mucocele of the middle turbinate.

soft tissue density within a grossly enlarged right concha bullosa, pushing the septum to the left side.

The patient was planned for endoscopic resection of the right concha. After resection of the lateral wall of the concha, a mass covered with nasal mucosa was identified occupying the concha, which was filled with mucus discharge suggestive of mucocele of the middle turbinate. The whole of the mucosal lining of the mucocele and the lateral wall of the middle turbinate were removed. The mucosal lining of the middle turbinate was put back in place. Septoplasty was performed in the same operation. Postoperatively, patient's symptoms were relieved completely.

\section{CASE 2}

A 27-year-old female patient presented with complaints of progressive bilateral nasal obstruction (right $>$ left) for 1 year, which was persistent and not relieved with medications. There was no history of nasal discharge, postnasal drip, headache or any other ENT complaint.

Anterior rhinoscopy revealed midline septum with bilateral enlarged middle turbinate, more on the right side. Nasal endoscopy confirmed the presence of bilateral concha bullosa (right $>$ left). There was a slight deviated nasal septum to the left side with right-sided spur.

CT scan of the nose and paranasal sinuses (Figure $2 \mathrm{~A}, \mathrm{~B}$ ) revealed a homogenous soft tissue density filling bilateral concha, right larger than left, as well as in bilateral ethmoid sinuses. A rightsided spur and mild left-sided septal deviation were also present.

The patient was planned for bilateral endoscopic conchoplasty, under general anaesthesia. On both sides, resection of the lateral bony wall revealed a mucosal-lined mass within the concha filled with mucus discharge, suggestive of mucocele of the middle turbinate. The mucosal lining of the mucocele and the lateral wall of the middle turbinate were removed. Mucosal lining of the middle turbinate was reposted back. The patient's postoperative evolution was uneventful.

\section{DISCUSSIONS}

Mucoceles are uncommon benign cystic lesions of the paranasal sinuses. The term mucocele refers to a cystic structure without the epithelium lining, which is usually caused by trauma. However, in the case of paranasal sinuses, they represent true mucus retention cysts, completely lined by respiratory epithelium and usually caused by obstruction of the sinus outflow tract ${ }^{3}$.

Concha bullosa, first described by Zuckerkandl in 1893, refers to pneumatised middle turbinate. This concha has an air cell lumen lined by respiratory ciliated epithelium with an ostium that connects it to the frontal recess. As such, inflammatory pathologies of the frontal recess area may rarely 

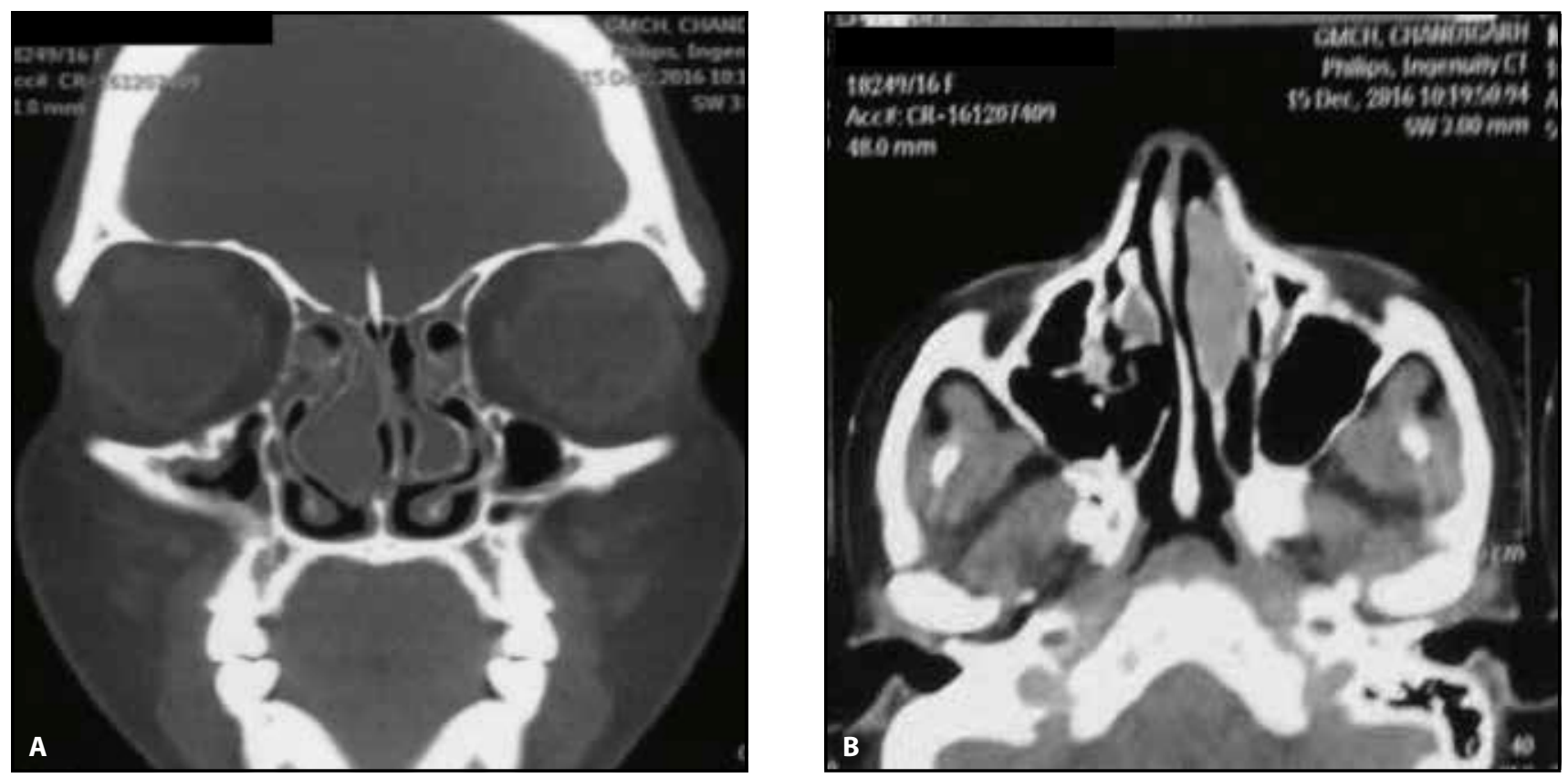

Figure 2. Coronal (A) and axial (B) sections of Computed tomography of a patient with bilateral concha bullosa (right > left) with homogenous soft tissue density within it, suggestive of mucocele of the middle turbinate.

impair aeration of the concha and predispose to mucocele formation.

Mucoceles are expansile lesions with the most common age of presentation between the fourth and seventh decade. Children and adolescents are rarely affected. However, they can be predisposed to mucocele formation especially with concomitant cystic fibrosis. Both males and females are equally affected ${ }^{4}$.

Mucoceles develop as a result of impaired drainage of the sinus, which can either be caused by alteration in the viscosity or composition of mucus as seen in cystic fibrosis or by mechanical obstruction in normal drainage of the sinus; these can be caused by some anatomical defects of the infundibulum, past trauma, recurrent and preceding rhinosinusitis, previous surgeries or benign and malignant tumors blocking the outflow. Obstruction of the normal drainage leads to an increased pressure in the sinuses and initiate an inflammatory cascade determined by the prostaglandins (prostaglandin E2) release and other inflammatory mediators secreted by the hyperplastic mucosa, leading to activation of osteoclasts and progression of the mucocele at the cost of erosion of the surrounding bone $\mathrm{e}^{5}$.

The frontoethmoidal complex is the most common site of mucocele formation owing to the anatomy of its outflow tract. Less frequently, mucoceles of the maxillary sinus, sphenoethmoidal area and isolated sphenoid sinus can also be observed. Owing to their pathophysiology, mucoceles can also be rarely seen in abnormally aerated structures in relation to the paranasal sinuses, such as the concha bullosa, clinoid process, pterygoid process and in areas of abnormally displaced mucosa, especially post trauma ${ }^{6}$.

Depending on the site and size, mucoceles have variable clinical presentation. The small ones may be asymptomatic, whilst large mucoceles may present with facial pain, headache, facial pressure, dental pain, ophthalmological alterations, and dacrocystitis ${ }^{7}$. Retroorbital headache, ocular muscle paresis, exophthalmos and optic neuropathy may be seen within sphenoidal or rarely anterior clinoid mucoceles ${ }^{1}$.

Mucoceles of the middle turbinate, since their first description by Badia et al. in 1994, have been associated with nasal obstruction, nasal discharge, diplopia, exophthalmos and chronic sinusitis symptoms owing to the blockage of ethmoidal infundibulum ${ }^{8}$.

Computed Tomography of the nose and paranasal sinuses is the imaging modality of choice. It shows homogenous soft tissue density within a sinus cavity with 10-18 HU attenuation, confirming the mucoid content, often increased to $20-40 \mathrm{HU}$ because of increased proteinaceous content, along with rounding of the bony lining as a result of bony remodelling secondary to expansion and bone erosion. Contrast enhancement, if done, is limited to the lining membrane. MRI is indicated when the diagnosis is uncertain or in cases of mucocele for- 
mation secondary to neoplasms. Mucoceles generally have low T1 and high T2 intensity; however, it may vary depending upon the content of the cyst ${ }^{9}$.

Progressive expansion of mucocele leads to thinning of the bone and pressure symptoms. As such, surgical management is deemed necessary to alleviate the symptoms. Simple drainage and marsupialization of the mucocele is the preferred procedure. Depending on the site, mucocele is marsupialized after widening the ostium, contents are drained, and the stent may be kept till the tract is epithelised. Mucosa lining the sinus needs not be removed. Aeration of the cavity thus reduces the risk of recurrence ${ }^{10}$.

Our cases illustrate a rare site of mucocele formation as the middle turbinate, which led to progressive nasal obstruction. Endoscopic surgery was performed and, post operatively, patients were relieved of symptoms.

\section{CONCLUSIONS}

Mucocele of the middle turbinate, although rarely, may present as a cause of unilateral or bilateral nasal obstruction, and therefore, must be kept into consideration especially when associated with other nasal or ocular complaints. The peculiarity of mucocele of the middle turbinate lies more in the diagnosis than in the management as most of these can be completely resected or marsupialized endoscopically, as seen in our cases.

Contribution of authors: All contributors meet the criteria for authorship.

Acknowledgements: None

Conflicting of interests: The author(s) declare that there is no conflict of interest.

Funding: None

\section{REFERENCES}

1. Selvakumar A, Mahalaxmi B, Ananth V, Gautam C. Sphenoidal mucocele causing bilateral optic neuropathy and ophthalmoplegia. Indian J Ophthalmol. 2014;62(4):515-7. DOI: 10.4103/0301-4738.99863.

2. Kandemir YB, Ergin I, Kandemir S, Sindel M. Bilateral middle concha bullosa mucopyocele connecting to headache disablement: a case study. Int J Anat Var. 2017;10(S1):75-6.

3. Toledano A, Herraiz C, Mate A, Plaza G, Aparicio JM, De Los Santos G, et al. Mucocele of the middle turbinate: a case report. Otolaryngol Head Neck Surg. 2002;126(4):442-4.

4. Gur OE, Kaymakci M, Sonbay Yilmaz DN. Paranasal sinus mucoceles. J Ann Eu Med. 2016;4(3):87-92. DOI: 10.4328/AEMED.97.

5. Rinna C, Cassoni A, Ungari C, Tedaldi M, Marianetti TM. Fronto - orbital mucoceles: our experience. J Craniofac Surg. 2004;15(5):885-9. DOI: 10.1097/00001665-200409000-00039.

6. Serrano E, Klossek JM, Percodani J, Yardeni E, Dufour X. Surgical management of paranasal sinus mucoceles: a long-term study of 60 cases. Otolaryngol Head Neck Surg. 2004;131(1):133-140. DOI: 10.1016/j. otohns.2004.02.014.

7. De Carvalho BV, Lopes ICC, Correa JB, Ramos LFM, Motta EGPC, Diniz RLFC. Typical and atypical presentations of paranasal sinus mucocele at computed tomography. Radiol Bras. 2013;46(6):372-5. DOI: 10.1590/ S0100-39842013000600010

8. Pinto JA, Cintra PPVC, de Marqui ACS, Perfeito DJP, Ferreira RDP, da Silva RH. Mucopyocele of the middle turbinate: a case report. Braz J Otorhinolaryngol. 2005;71(3):378-81. DOI: 10.1016/s18088694(15)31340-9.

9. Lloyd G, Lund VJ, Savy L, Howard D. Optimum imaging for mucoceles. J Laryngol Otol. 2000;114(3):233-6. DOI: 10.1258/0022215001905238.

10. Har-El G, Balwally AN, Lucente FE. Sinus mucoceles: is marsupialisation enough? Otolaryngol Head Neck Surg. 1997;117(6):633-40. DOI: 10.1016/s0194-5998(97)70045-7. 